JOURNAL of

MAINE MEDICAL CENTER Journal of Maine Medical Center

\title{
2021 Lambrew Research Retreat; Thomas Maciag Award for Excellence in Basic and Translational Science
}

Follow this and additional works at: https://knowledgeconnection.mainehealth.org/jmmc

\section{Recommended Citation}

(2021) "2021 Lambrew Research Retreat; Thomas Maciag Award for Excellence in Basic and Translational Science," Journal of Maine Medical Center. Vol. 3 : Iss. 2 , Article 18.

Available at: https://knowledgeconnection.mainehealth.org/jmmc/vol3/iss2/18 https://doi.org/10.46804/ 2641-2225.1112

The views and thoughts expressed in this manuscript belong solely to the author[s] and do not reflect the opinions of the Journal of Maine Medical Center or MaineHealth.

This Supplement is brought to you for free and open access by Maine Medical Center Department of Medical Education. It has been accepted for inclusion in the Journal of Maine Medical Center by an authorized editor of the MaineHealth Knowledge Connection. For more information, please contact Dina McKelvy mckeld1@mmc.org.

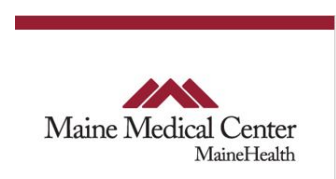




\section{Primary cilia of mammalian cardiomyocytes:}

\section{Potential role in development of the ventricular myocardium?}
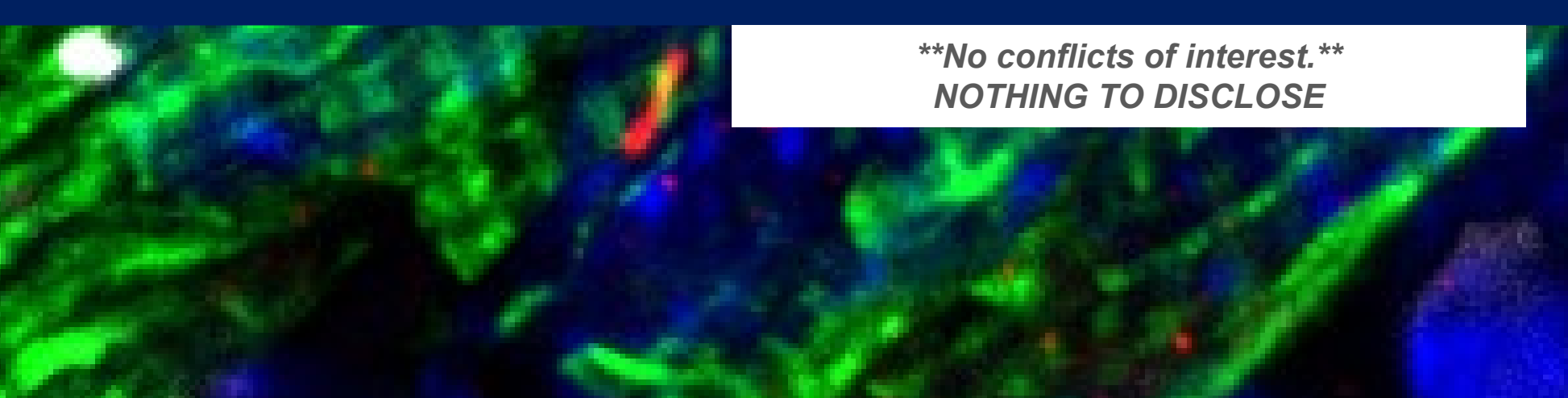

No conflicts of interest.** NOTHING TO DISCLOSE

\section{ra}

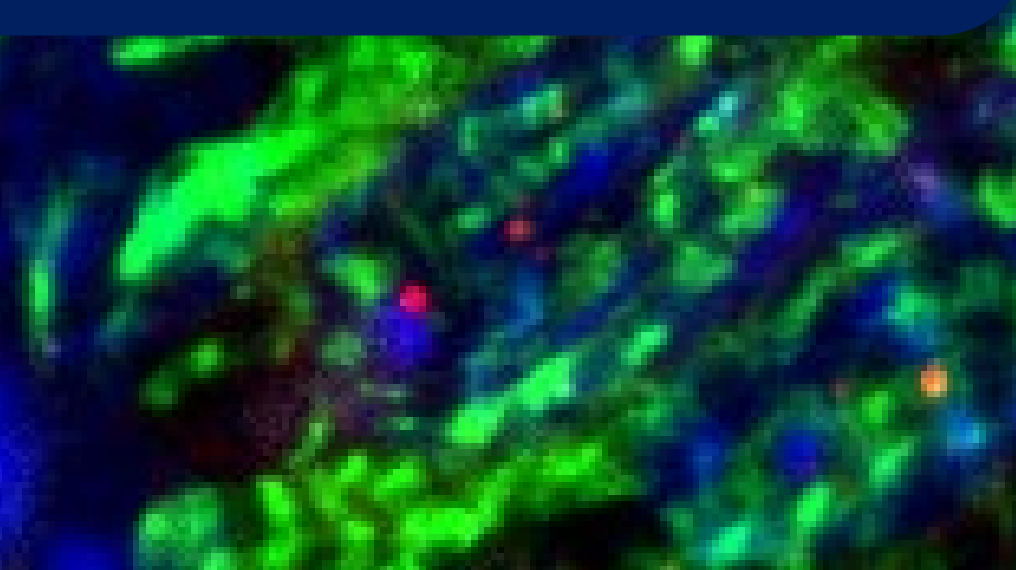

Lindsey Avery Fitzsimons, MS, PhD Candidate

K. L. Tucker Laboratory

Department of Biomedical Sciences

University of New England College of Osteopathic Medicine

Biddeford, Maine

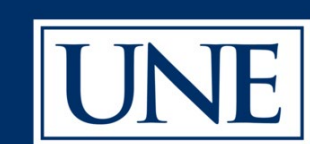

UNIVERSITY OF NEW ENGLAND 


\section{The Primary cilium: A CELLULAR ORGANELLE}
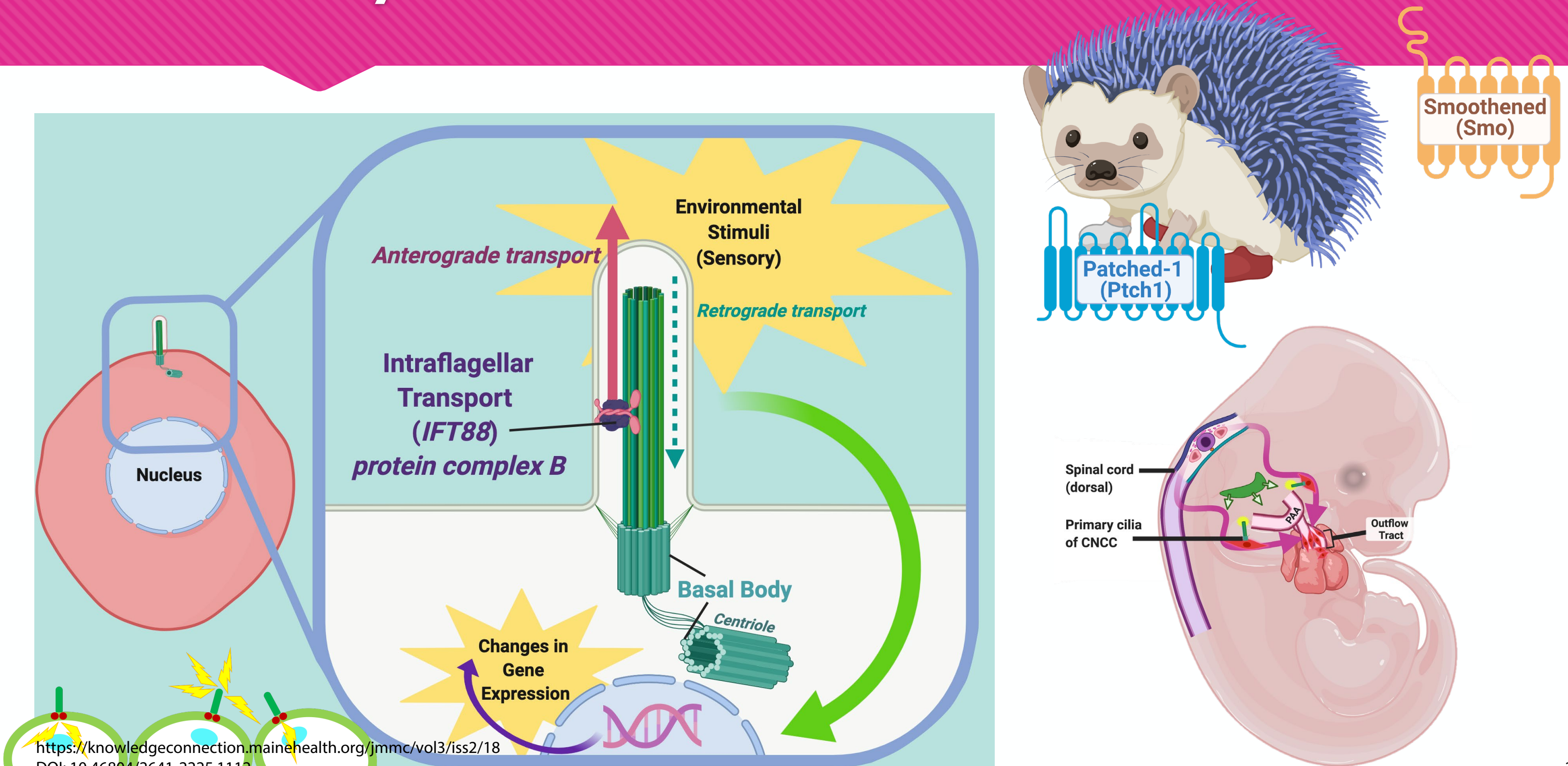

(dorsal)

Primary cilia of CNCC

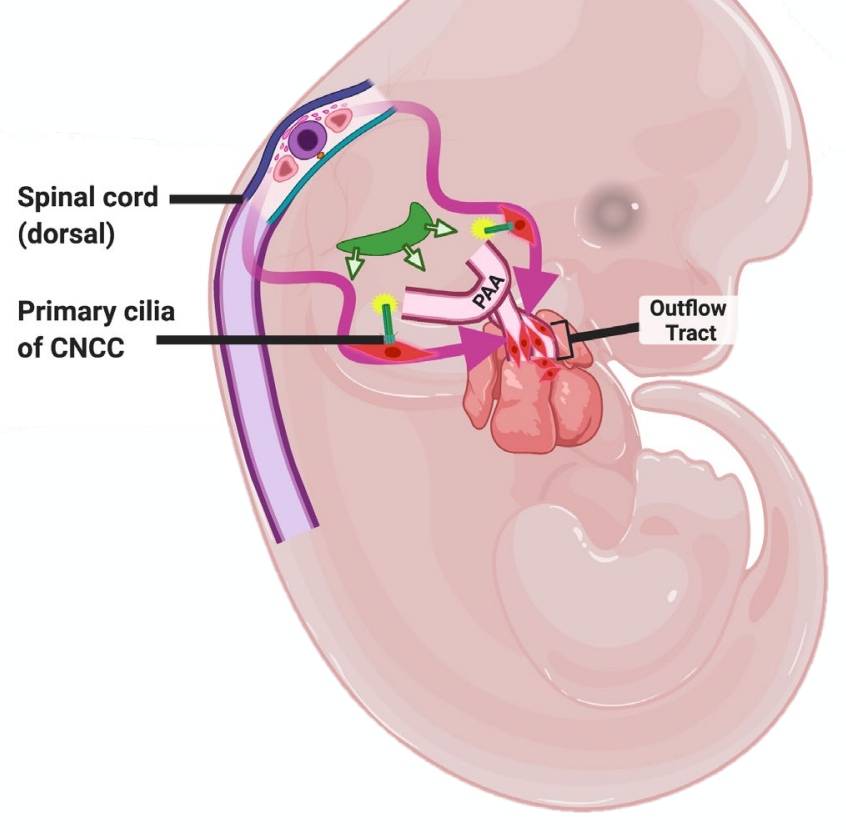




\section{Primary Cilia of Cardiomyocyies}

\section{Are they relevant beyond development?}

SCIENCE TRANSLATIONAL MEDICINE | RESEARCH ARTICLE

\section{CARDIOVASCULAR DISEASE \\ Primary cilia defects causing mitral valve prolapse \\ Katelynn A. Toomer ${ }^{1 *}$, Mengyao $\mathrm{Yu}^{2,3 *}$, Diana Fulmer', Lilong Guo', Kelsey S. Moore',

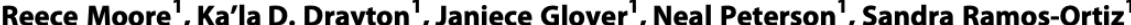 Alex Drohan ${ }^{1}$, Breiona J. Catching ${ }^{1}$, Rebecca Stairley ${ }^{1}$, Andy Wessels' ${ }^{1}$, Joshua H. Lipschutz ${ }^{4,5}$ Francesca N. Delling ${ }^{6}$, Xavier Jeunemaitre ${ }^{2,3,7}$, Christian Dina ${ }^{6,9}$ Harrison Brand ${ }^{10}$, Michael E. Talkowski ${ }^{10}$, Federica del Monte ${ }^{13}$, Rupak Mukherjee ${ }^{11}$

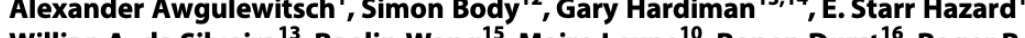 Wilian A. da Silveira 'Baolin Wang ${ }^{2}$, Maire Leyne ${ }^{2}$, Ronen Durst ', Roger R. Markwald', Solena Le Scouarnec, Albert Hagege ${ }^{20+}$, Thierry Le Tourneau ${ }^{80}$, Peter Kohh ${ }^{80}$, Eva A. Rog-Zielinska ${ }^{18}$ Patrick T. Ellinor ${ }^{19}$, Robert A. Levine ${ }^{2}$, David J. Milan ${ }^{19,21+}$, Jean-Jacques Schottt ${ }^{8,9+}$, Nabila Bouatia-Naji ${ }^{2,3+}$, Susan A. Slaugenhaupt ${ }^{10 \dagger}$, Russell A. Norris ${ }^{1+4}$}

PMCID: PMC6517085

Circulation. Author manuscript; available in PMC 2020 May 14 Published in final edited form as:

Circulation. 2019 May 14; $139(20)$ : 2342-2357.

NIHMSID: NIHMS1525148

doi: $10.1161 /$ CIRCULATIONAHA. 117.028752

\section{Fibroblast Primary Cilia are Required for Cardiac Fibrosis}

Elisa Villalobos, $\mathrm{PhD},{ }^{1,3, \dagger}$ Alfredo Criollo, $\mathrm{PhD},{ }^{1,3,4, \dagger}$ Gabriele G. Schiattarella, MD, PhD, ${ }^{1}$ Francisco Altamirano, $\mathrm{PhD},{ }^{1}$ Kristin M. French, PhD, ${ }^{1}$ Herman I. May, MSc, ${ }^{1}$ Nan Jiang, MSc, ${ }^{1}$ Ngoc Uyen Nhi Nguyen, PhD, ${ }^{1}$ Diego Romero, MSc, ${ }^{5}$ Juan Carlos Roa, MD, ${ }^{5}$ Lorena García, PhD, ${ }^{3}$ Guillermo Diaz-Araya, PhD, ${ }^{3}$

Eugenia Morselli, PhD, ${ }^{6}$ Anwarul Ferdous, $\mathrm{PhD},{ }^{1}$ Simon J. Conway, $\mathrm{PhD},{ }^{7}$ Hesham A. Sadek, MD, PhD, ${ }^{1}$ Thomas G. Gillette, PhD, ${ }^{1}$ Sergio Lavandero, $\mathrm{PhD},{ }^{1,3,{ }^{*}}$ and Joseph A. Hill, MD, PhD ${ }^{1,2,{ }^{*}}$

\section{Anterograde transport}

(Anterograde lengthening)

rights reserved;
exclusive licensee

American Association
for the Advancement

of Science. No claim

(a)

PMID: $\underline{30818997}$

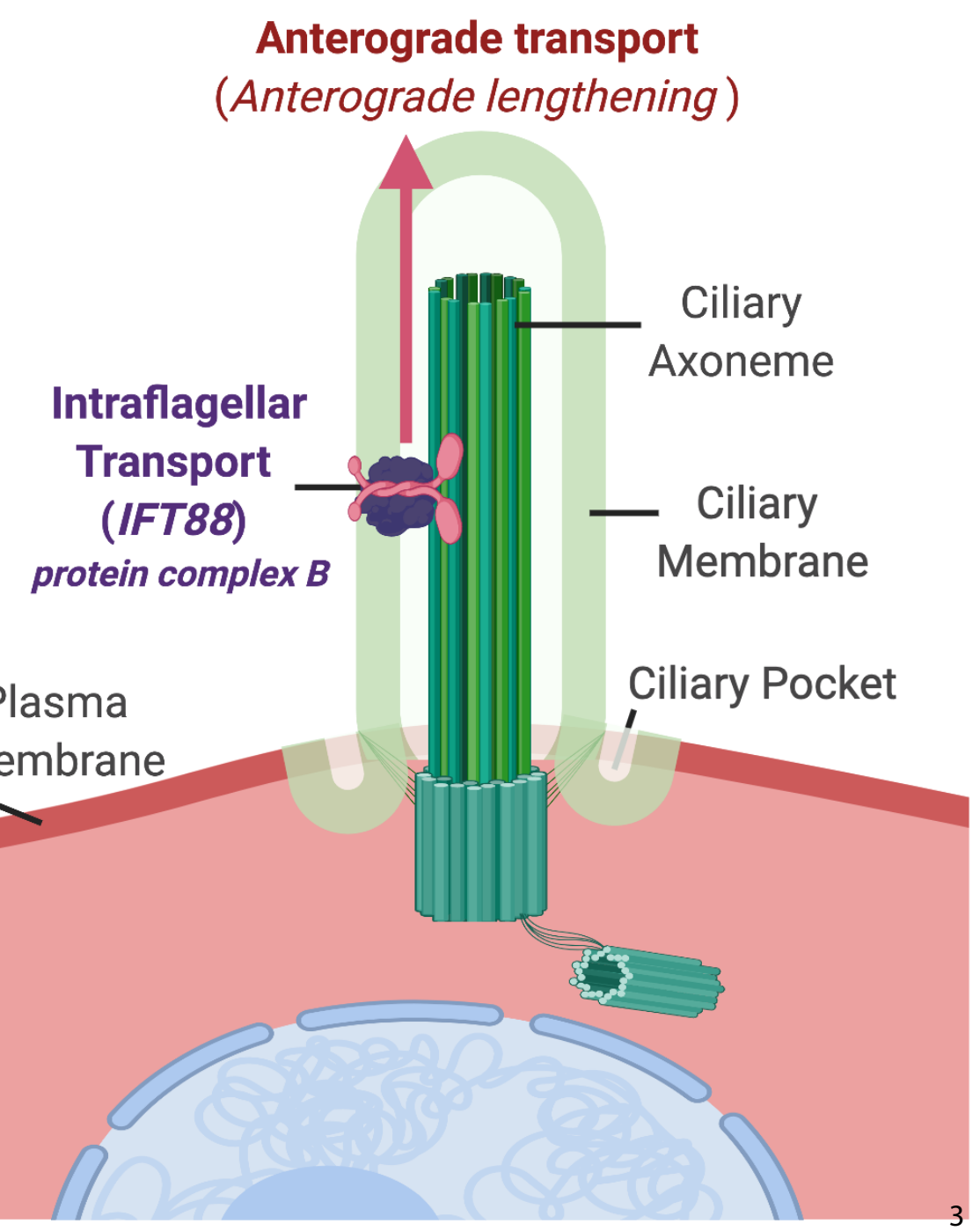




\section{Loss of primary cilia in embryonic CM}

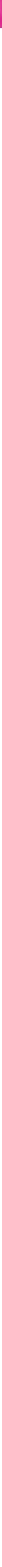




\section{In vivo loss of CM primmerty cilfie}

impairs myocardial maturation
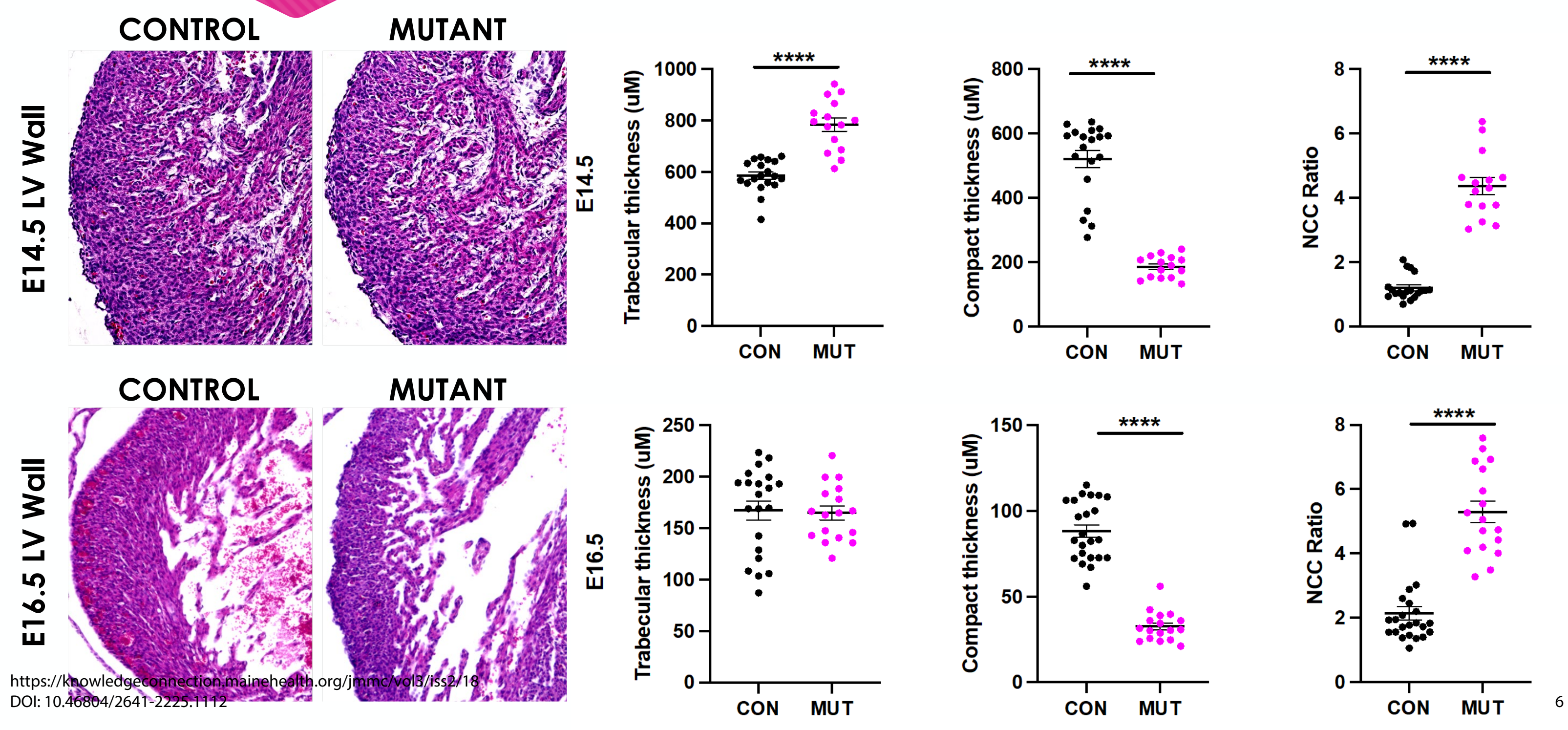


\section{Hedgehog GOF in developing CM}

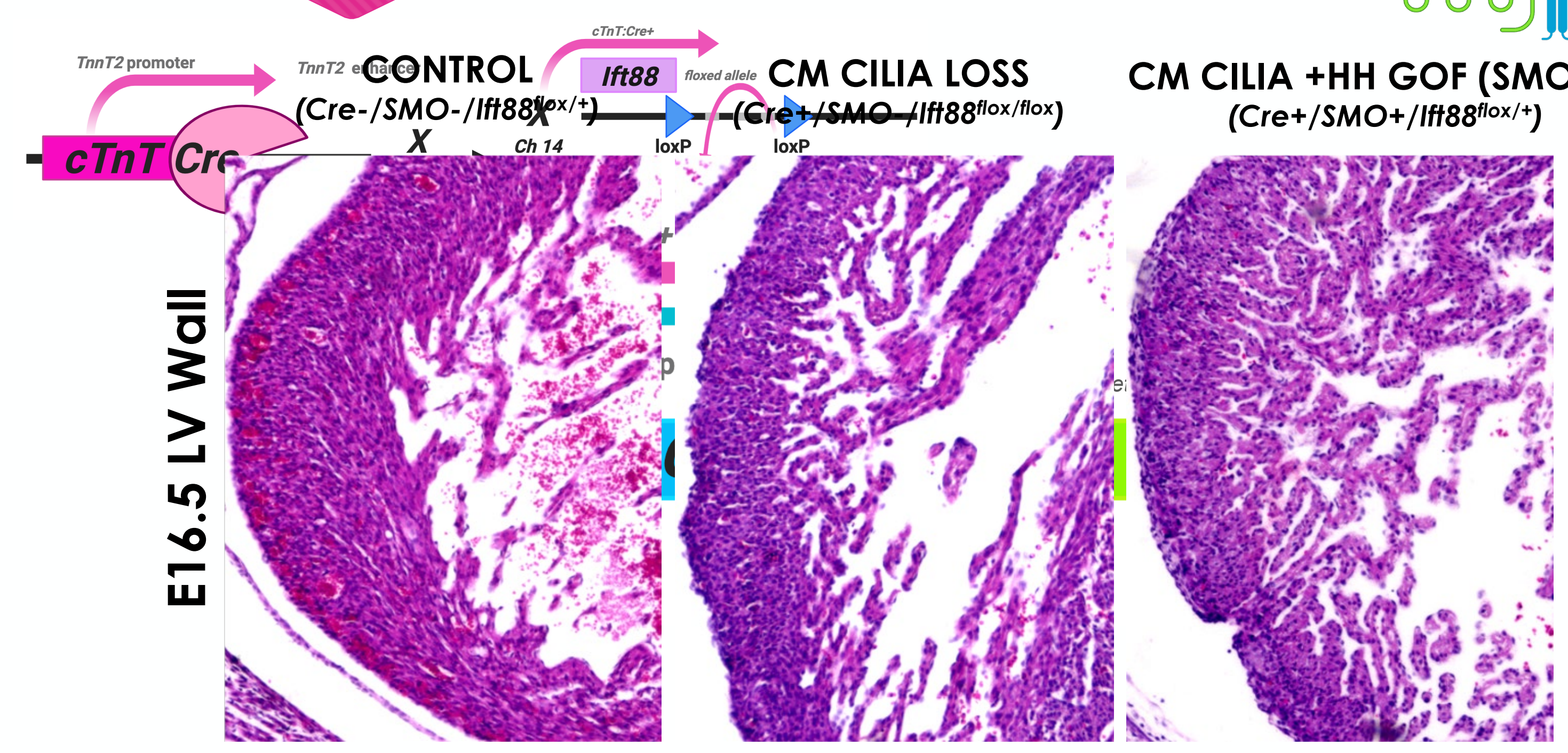




\section{Hedgehog GOF in developing CM}
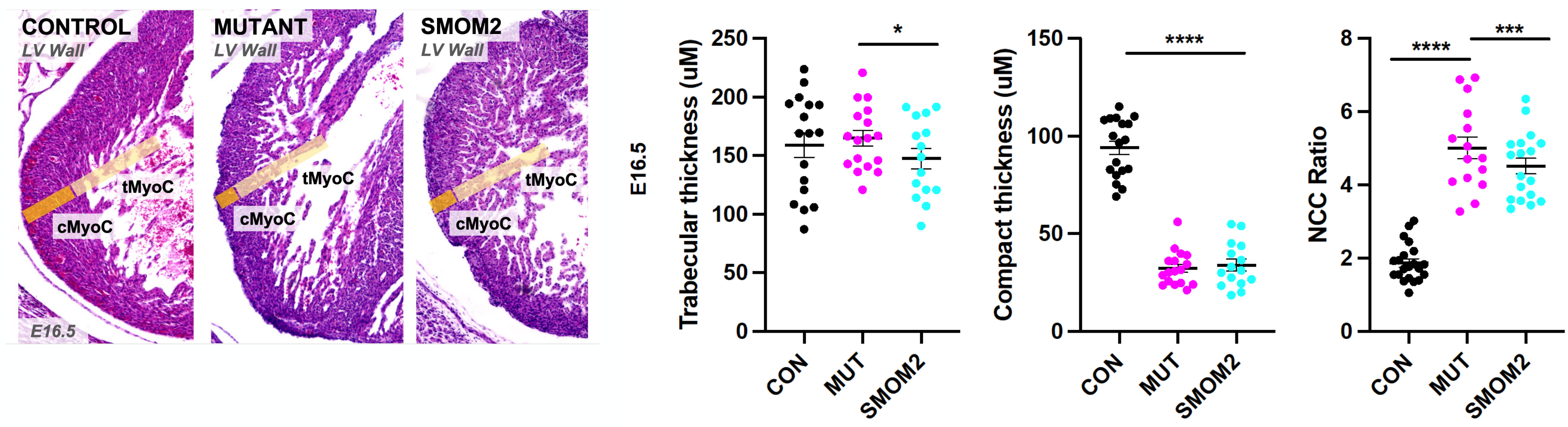


\section{CHD \& Myocardiet Phenoty pess Primary cillia in the pediatric heart with CHD}

Maine Medical Center Research Institute

The Maine Medical Center Biobank (MMC BB)

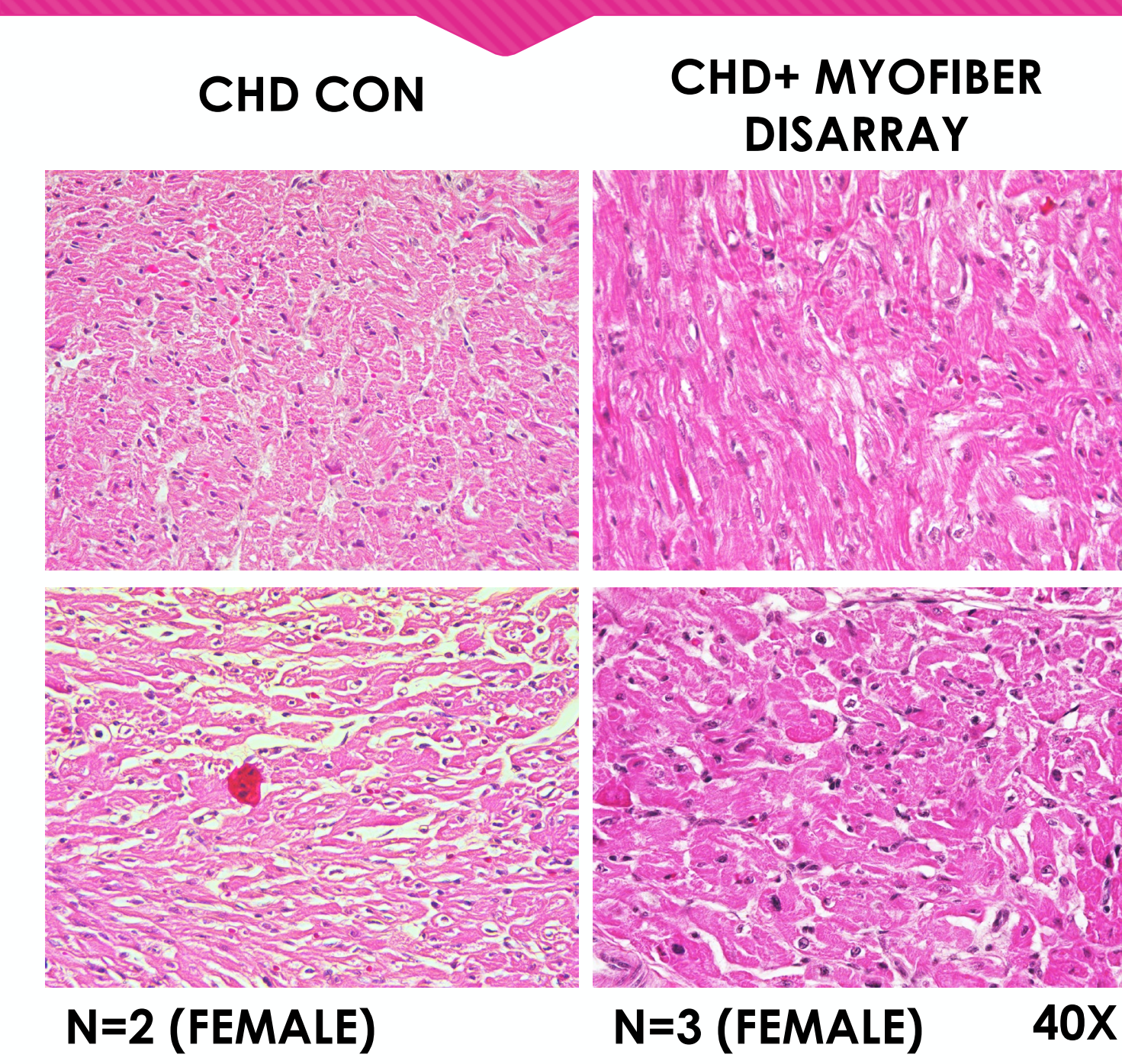

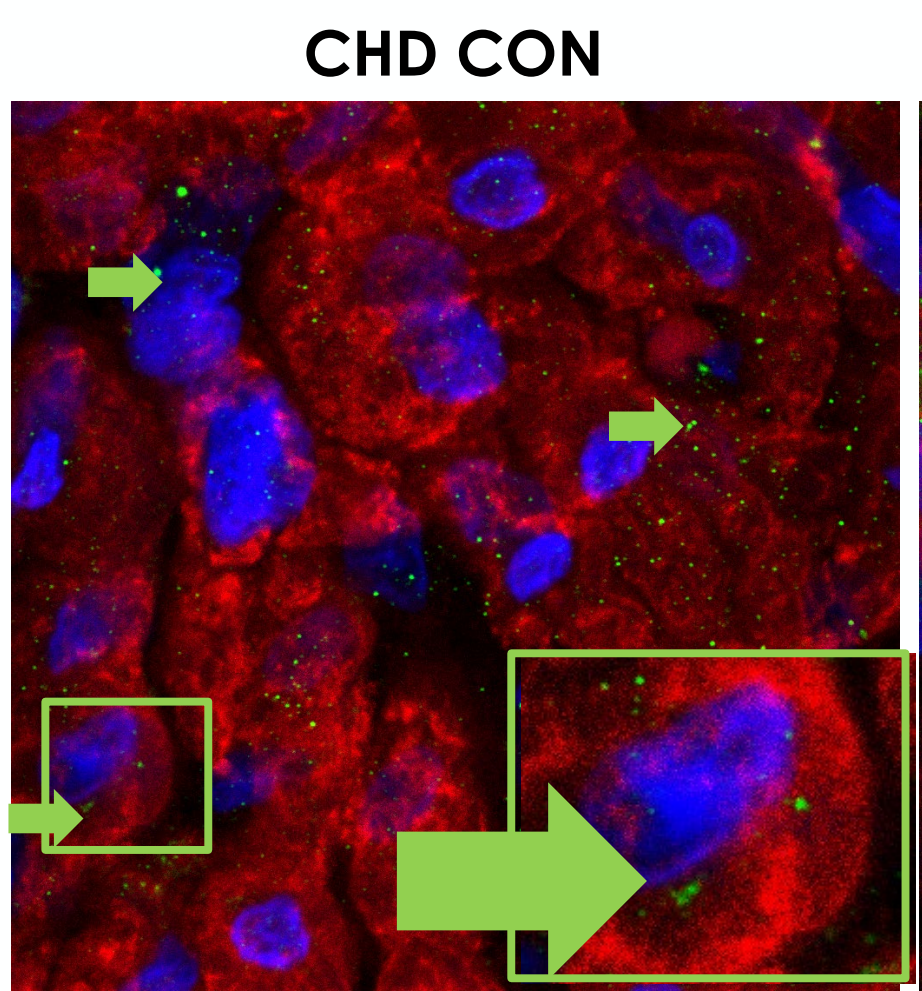

DAPI/Nuclei

$\alpha$ Cardiac Troponin-T (cTnT) /555

a ARL13B/Primary cilia/488

\section{CHD+ MYOFIBER DISARRAY}

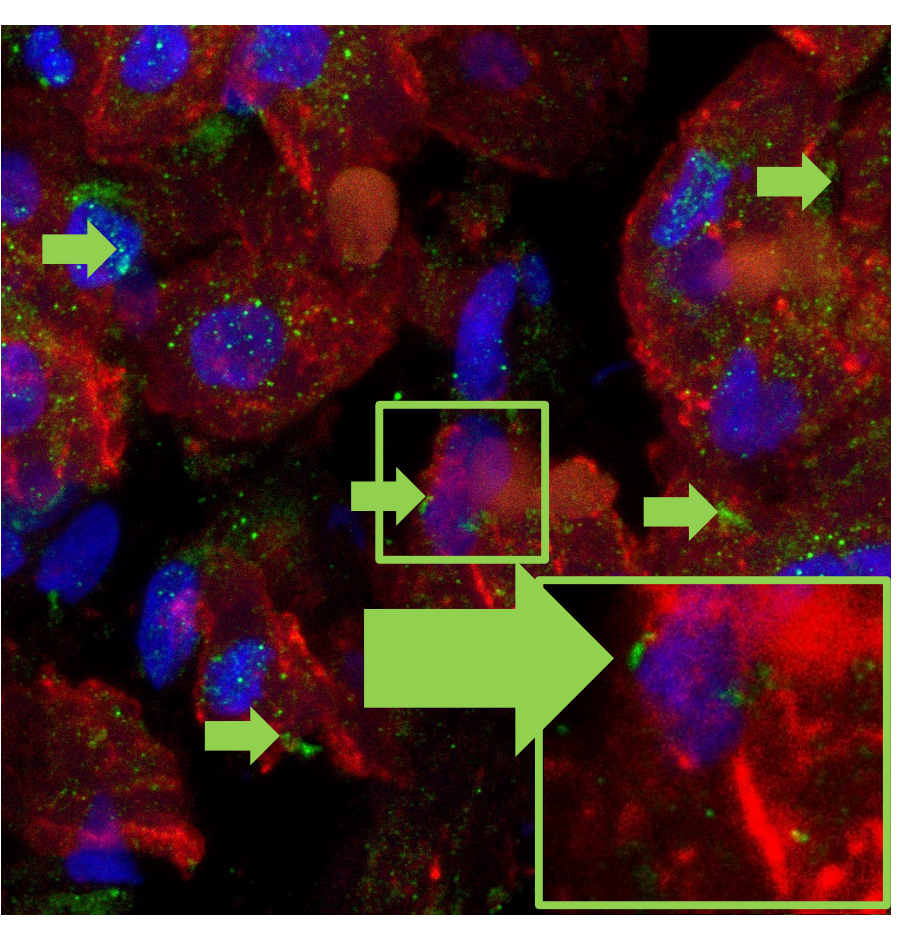

63X/Zoom 4.00 


\section{Ciliation in Myofiber Disarray \& CHD}
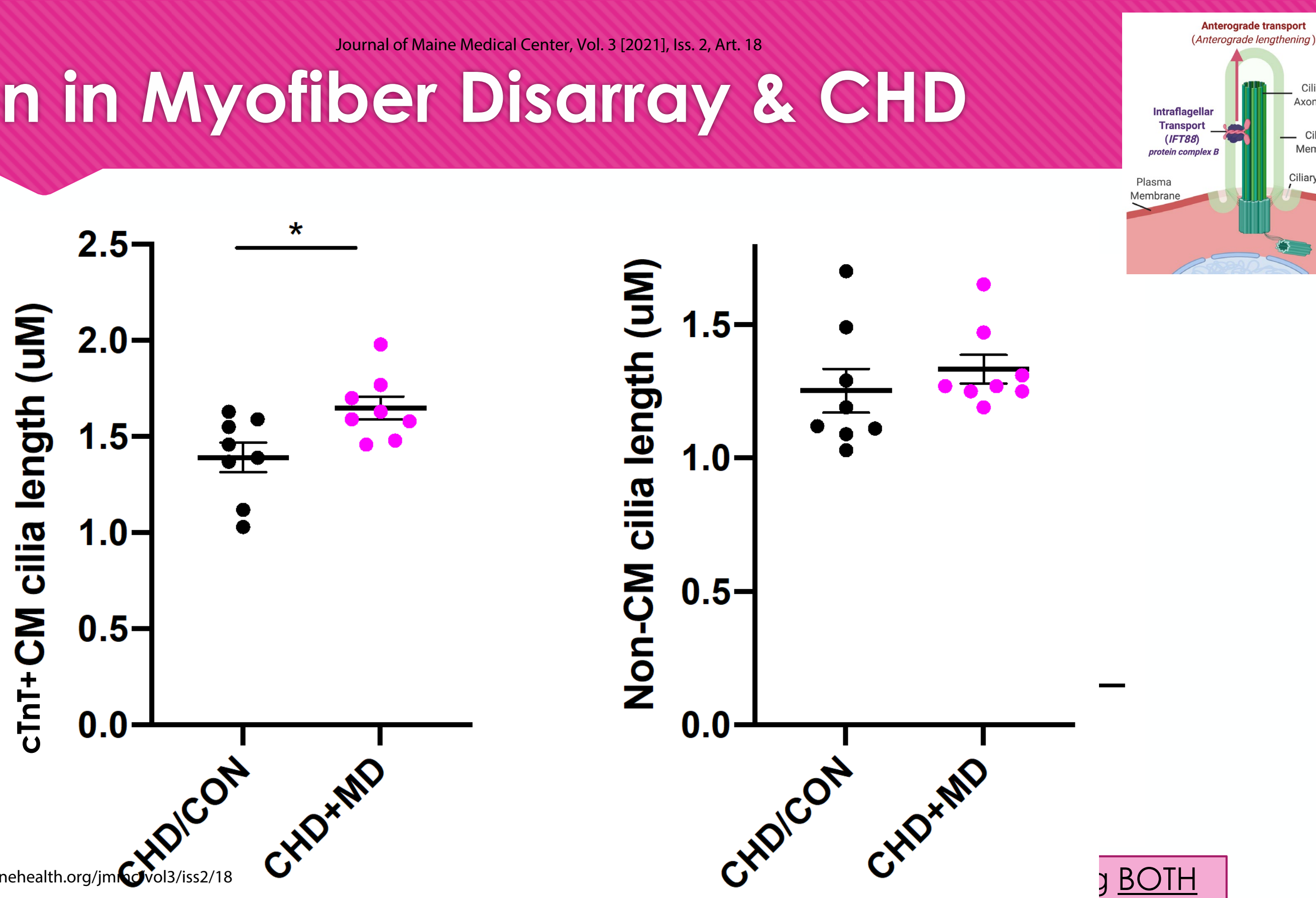


\section{Conclusions \& Future Directions}

- Primary cilia are present in embryonic (Mouse) and postnatal (Human), ventricular cardiomyocytes

- Loss of primary cilia in developing cardiomyocytes (Mouse) leads to sarcomere disorganization and noncompaction of the ventricular myocardium

Anterograde transport

- Human CHD with Myocardial Disarray is correlated with:

1) lengthening of primary cilia in cardiomyocytes

2) increased ciliation of cardiomyocytes

- Primary cilia represent a viable and modulatory target for improving our understanding of/treatment for CHD involving ventricular myocardium

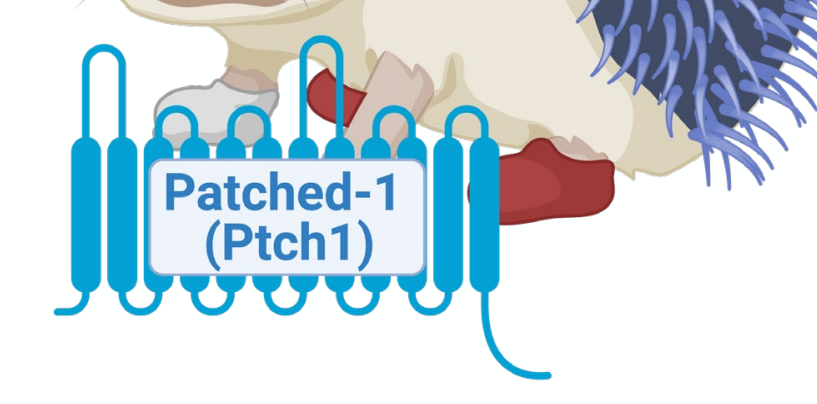

$$
\text { (Anterograde lengthening) }
$$




\section{Acknowledgemernts}

\section{TUCKER LABORATORY, UNE}

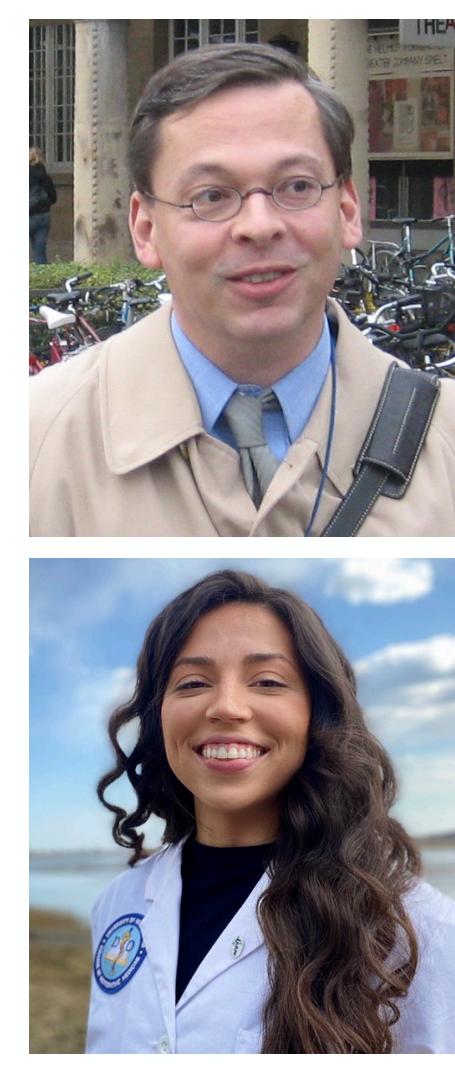

\section{Kerry L. Tucker, PhD}

Associate Professor

University of New England

College of Osteopathic Medicine

Dept. of Biomedical Sciences

$$
\begin{aligned}
& \text { BEST } \\
& \text { BOSS } \\
& \text { EVER }
\end{aligned}
$$

\section{Delanie Kneeland, BS}

\section{OMS-I}

University of New England

College of Osteopathic Medicine

\section{PEDIATRIC DONOR SAMPLES}

The Maine Medical Center

Biobank (MMC BB)

Anne Breggia MMC BB Director

Biobank Team (MMC)

Maine Medical Center

Research Institute

UNE Histology \& COBRE Imaging Core

Peter Caradonna, BS
DISSERTATION COMITTEE

Tom Gridley, PhD MMCRI

\section{Lucy Liaw, PhD} MMCRI

Kristy Townsend, PhD Univ. of Maine The Ohio State University James Schwob, MD, PhD Tufts University

Manager, COBRE Histology and Imaging Core
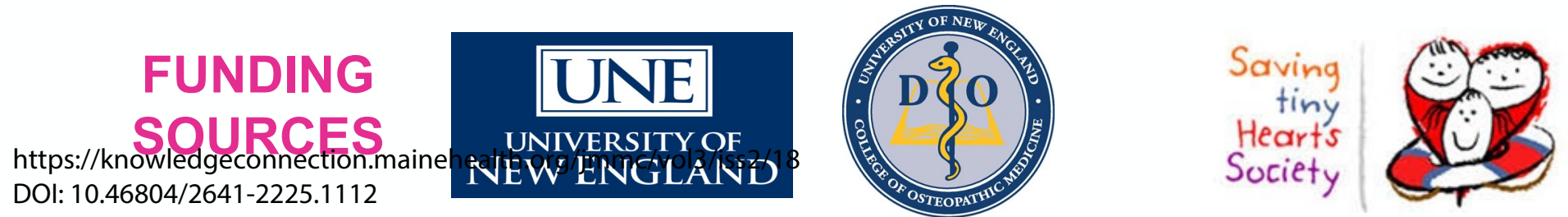


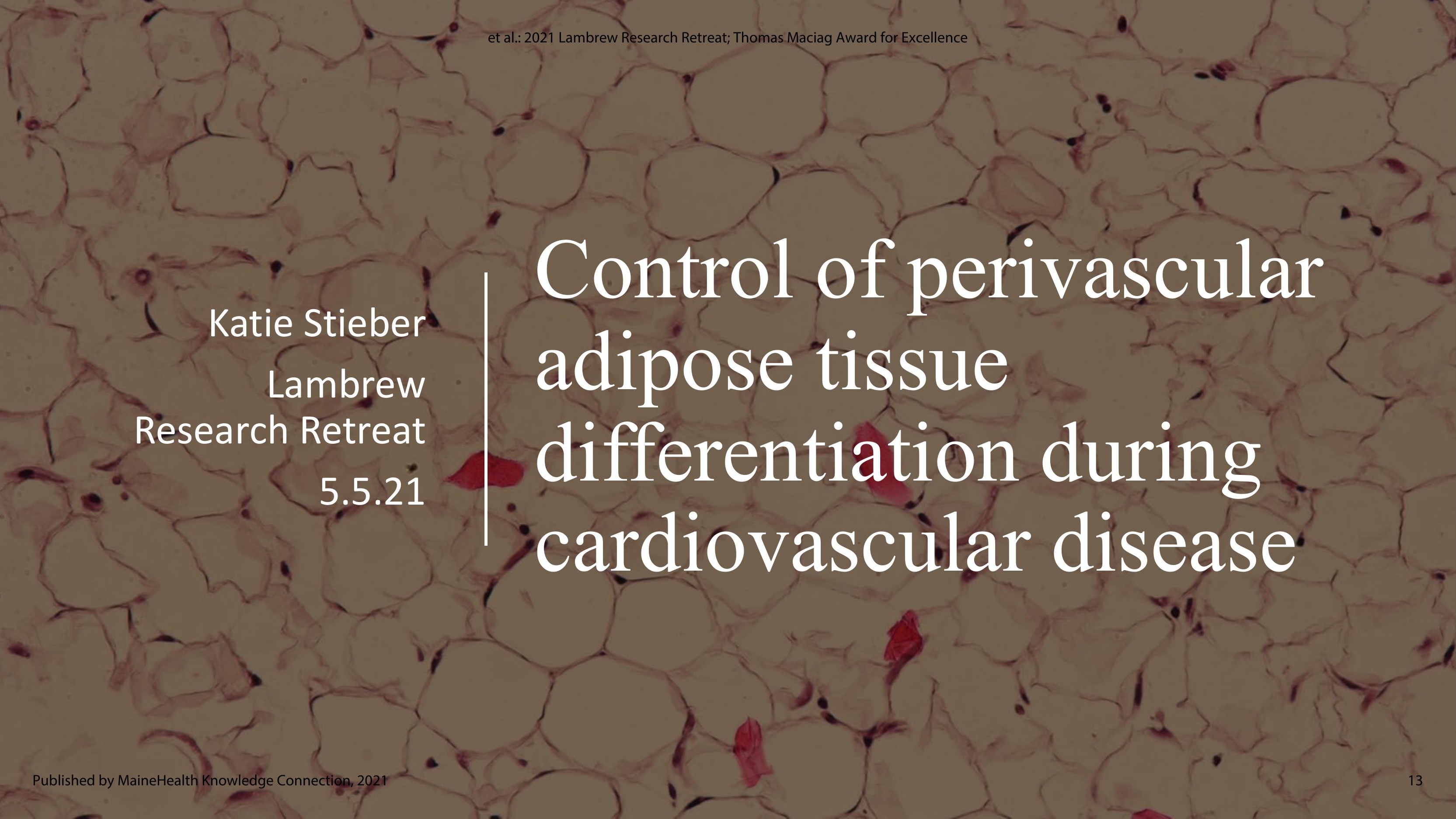




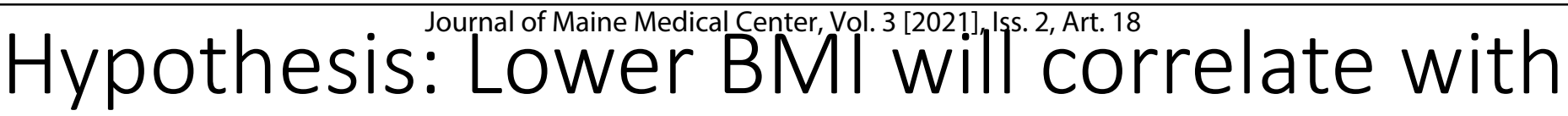 lower RAB27a expression}

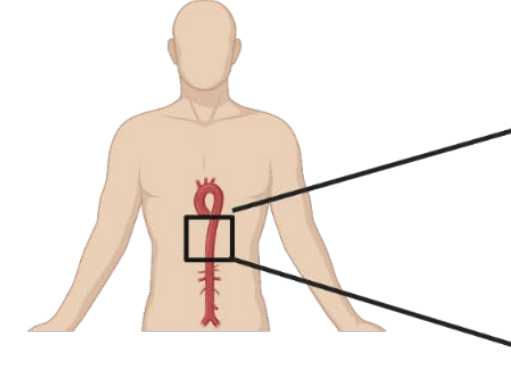

Valve repair

(VR)

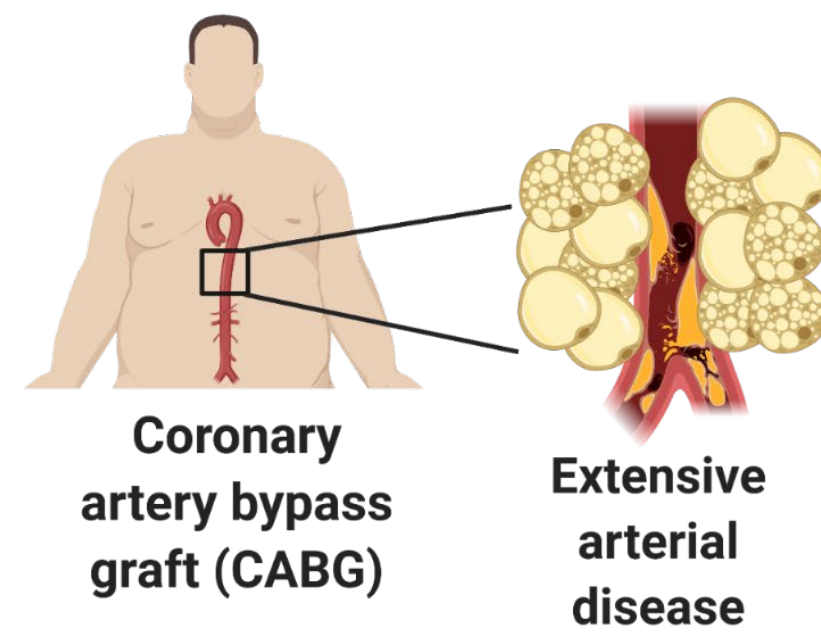

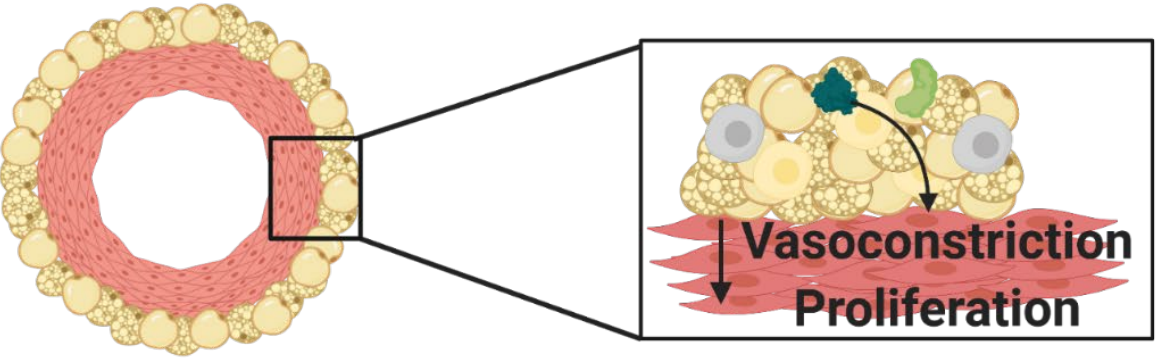

PVAT on

vessel cross section

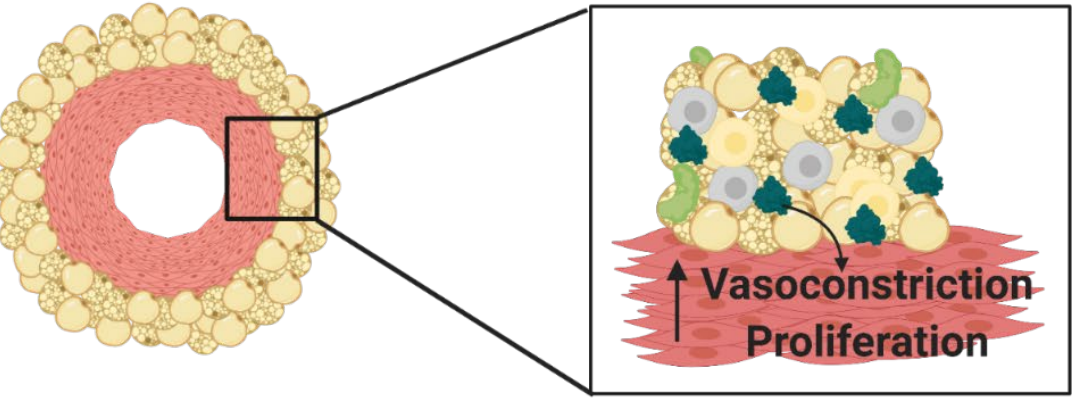

Expanded PVAT

on vessel cross

section 


\section{VR donors are physiologically different than CABG donors}

Average BMI

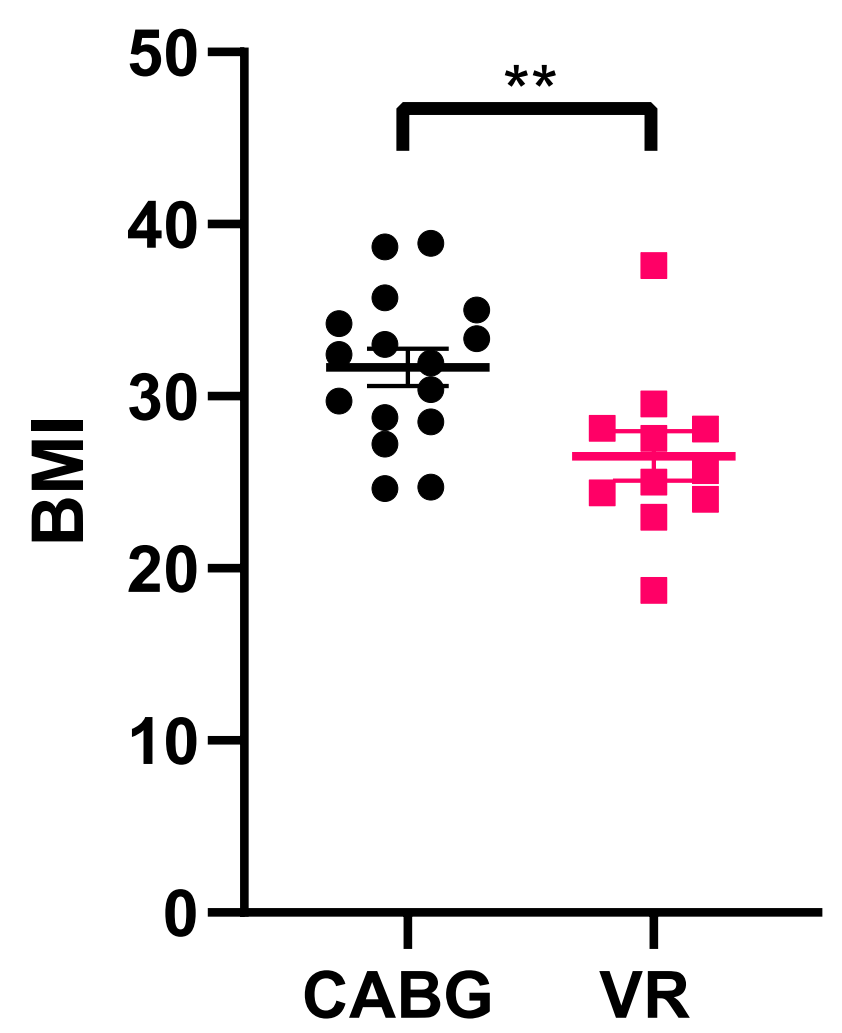

\section{Rab27a}

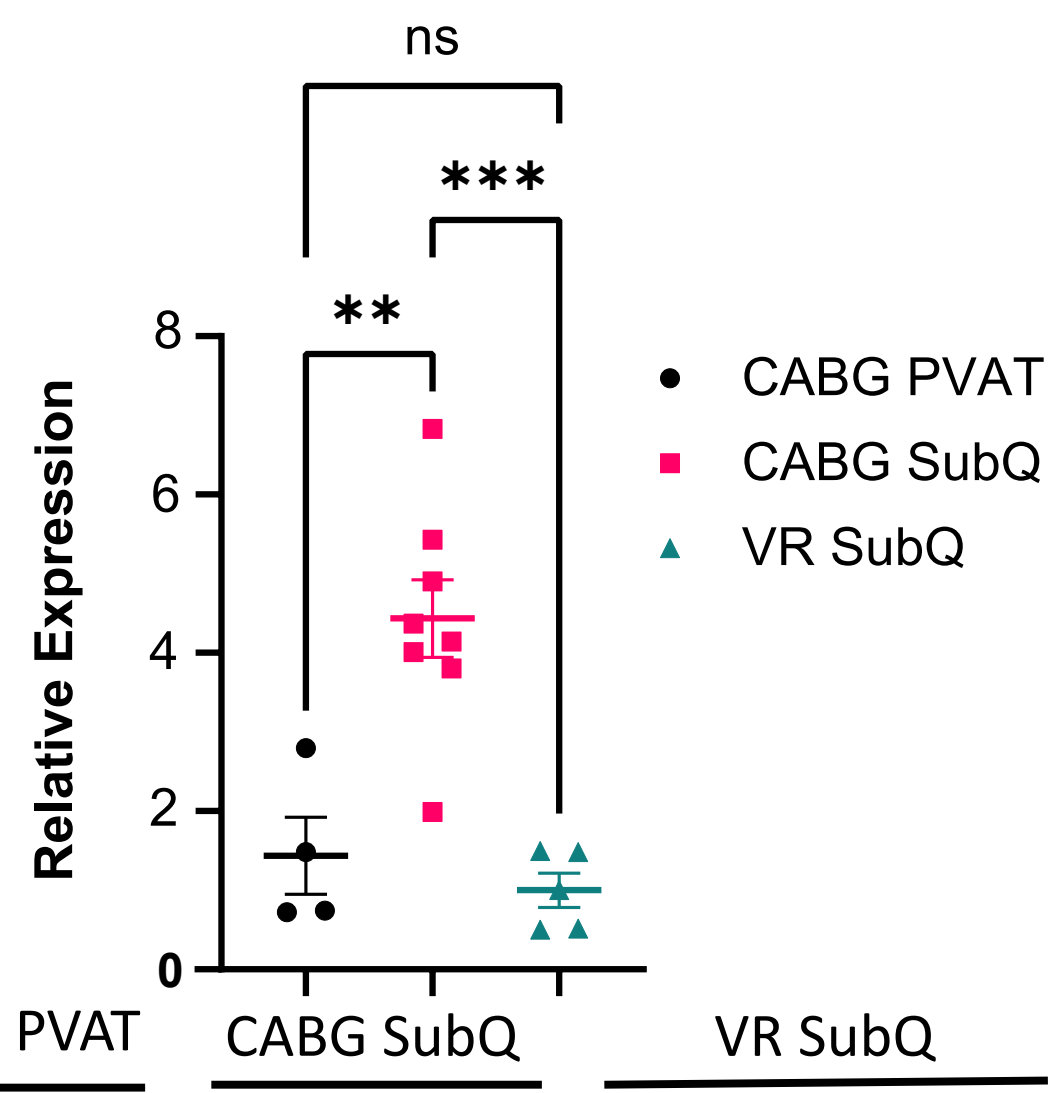

\begin{tabular}{|c|c|c|}
\hline & CABG & VR \\
\hline $\begin{array}{c}\text { Average } \\
\text { age }\end{array}$ & 70 & 65.45 \\
\hline $\begin{array}{c}\text { Donors } \\
\text { with } \\
\text { diabetes }\end{array}$ & $5 / 16$ & $0 / 11$ \\
\hline
\end{tabular}




\section{Acknowleđgrments and References}

The Liaw Lab

Ashley Soucy

Ben Tero

Cathleen Huang

Ginger Paquette

Kimberly Malka

Larisa Ryzhova

Lucy Liaw

Previous Members:

Joshua Boucher

Penny Clum

Jackie Turner

Jess Davis-Knowlton

Bethany Fortier

Michael Robich

\section{Committee Members}

Dr. Rob Koza

Dr. Lucy Liaw

Dr. Michaela Reagan

Dr. Cal Vary

Dr. Robert Burgess

Diagrams created with biorender.com

C. Stieber was supported by the University of Maine institutional training grant

1T32GM132006-01 from the National

Institute of General Medical Sciences (PIs: L.

Liaw and C. Henry), F31 HL156320-01 from

the National Heart, Blood, and Lung Institute, and R01 HL141149.

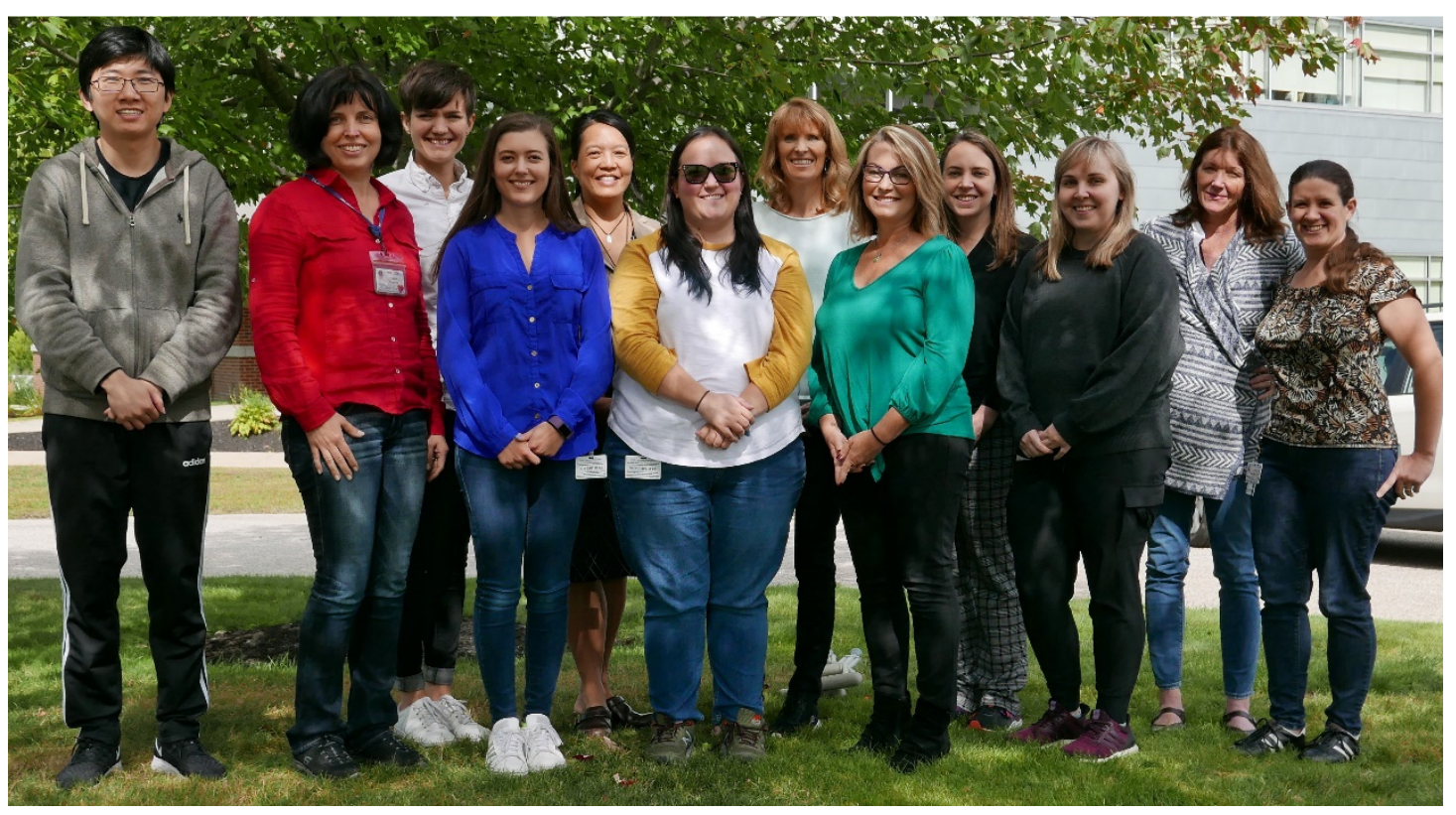

Email: caitlin.stieber@maine.edu Twitter: @Katie_Stieber LinkedIn: Caitlin Stieber
Maine Medical Center

https://knomedgrchnitecstitiththainehealth.org/jmmc/vol3/iss2/18 DOI: 10.46804/2641-2225.1112
GSBSE

Biomedical Science and Engineering 